\title{
Frontal white matter integrity in adults with Down syndrome with and without dementia
}

\author{
David Powell ${ }^{a}$, Allison Caban-Holt ${ }^{b}$, Gregory Jicha ${ }^{b, c}$, William Robertson ${ }^{c}$, Roberta Davis ${ }^{b}$, \\ Brian T. Golda, ${ }^{a}$, Frederick A. Schmitt ${ }^{\mathrm{b}, \mathrm{c}}$, and Elizabeth Head ${ }^{\mathrm{b}, \mathrm{e},{ }^{*}}$ \\ aMagnetic Resonance Imaging and Spectroscopy Center, University of Kentucky, Lexington, KY, \\ USA \\ bSanders-Brown Center on Aging, University of Kentucky, Lexington, KY, USA \\ 'Department of Neurology, University of Kentucky, Lexington, KY, USA \\ dDepartment of Anatomy and Neurobiology, University of Kentucky, Lexington, KY, USA \\ eDepartment of Molecular and Biomedical Pharmacology, University of Kentucky, Lexington, KY, \\ USA
}

\begin{abstract}
Adults with Down syndrome (DS) are at high risk for developing Alzheimer's disease after the age of 40 years. To detect white matter (WM) changes in the brain linked to dementia, fractional anisotropy (FA) from diffusion tensor imaging was used. We hypothesized that adults with DS without dementia (DS $n=10)$, DS with dementia (DSAD $n=10)$ and age matched non-DS subjects (CTL $n=10$ ) would show differential levels of FA and an association with scores from the Brief Praxis Test and the Severe Impairment Battery. WM integrity differences in DS compared with CTL were found predominantly in the frontal lobes. Across all DS adults, poorer Brief Praxis Test performance correlated with reduced FA in the corpus callosum as well as several association tracts, primarily within frontoparietal regions. Our results demonstrate significantly lower WM integrity in DS compared with controls, particularly in the frontal tracts. DS-related WM integrity reductions in a number of tracts were associated with poorer cognition. These preliminary results suggest that late myelinating frontal pathways may be vulnerable to aging in DS.
\end{abstract}

\section{Keywords}

Aging; Brief praxis test; BPT; Dementia questionnaire for persons with; mental retardation; DMR; Diffusion tensor imaging; Fractional anisotropy; Severe impairment battery; SIB; Trisomy 21

\footnotetext{
(C) 2014 Elsevier Inc. All rights reserved.

*Corresponding author at: University of Kentucky, Sanders-Brown Center on Aging, 203 Sanders-Brown Building, 800 South Limestone Street, Lexington, KY, 40515, USA. Tel.: +1 859257 1412×481; fax: 859-323-2866. Elizabeth.head@uky.edu (E. Head). Elizabeth Head and Frederick A. Schmitt are co-senior authors.

Disclosure statement

The authors have no actual or potential conflicts of interest.

All human subjects research was approved by the University of Kentucky Institutional Review Board.
} 


\section{Introduction}

The most common cause of Down syndrome (DS) is triplication of chromosome 21, resulting in a phenotype that is accompanied by altered brain development and other neurologic features (Lott, 2012). However, a key challenge for adults with DS as they age is the increasing risk for developing Alzheimer's disease (AD). Despite estimated ages of dementia onset in DS of 48-56 years (reviewed in Head et al., 2007; Schupf and Sergievsky, 2002), AD neuropathology appears in virtually all adults with trisomy 21 after the age of 40 years (Wisniewski et al., 1985). Thus, there may be up to a 10-year delay in the onset of clinical symptoms of dementia and the presence of AD neuropathology, as has been suggested for late onset sporadic $\mathrm{AD}$ in the general population.

Diffusion tensor imaging (DTI) represents a noninvasive in vivo method for characterizing the microstructural properties of white matter (WM) by measuring the rate and direction of diffusion of water molecules in the neural tissue (Basser et al., 2000) that can occur equally (isotropic) or unequally (anisotropic) in all directions. Anisotropy in WM indicates disruption in WM integrity resulting from a loss of compactness of WM tracts, their myelination, and/or number of axons within the tract studied (Wimberger et al., 1995). Fractional anisotropy (FA) measures these changes in ranges from 0 (diffusion that is equal in all directions representing poor white matter integrity) to 1 (diffusion that is predominately in one direction representing good white matter integrity) (Pfefferbaum and Sullivan, 2003).

DTI has been used extensively to study both brain aging and disease states such as AD (Sexton et al., 2011). Results from several studies have suggested that FA decreases are associated with age-related declines on memory and executive control tasks (Bucur et al., 2008; Gold et al., 2010a, 2010b). Studies of normal aging have consistently reported decreases in FA, suggesting a loss in WM integrity inherent in the aging process, that is amplified in disease states such as AD (Madden et al., 2012). Most DTI studies of aging and dementia have reported that age-related FA declines follow an anterior-posterior gradient with WM in frontal regions showing the earliest and largest declines are associated with executive decline evident on neuropsychological testing (Madden et al., 2009).

In DS, there is a growing literature suggesting that the earliest manifestations of dementia appear to involve changes in personality and behavior (Aylward et al., 1997; Cooper and Prasher, 1998; Holland et al., 2000), which are likely frontal-dependent. Pragnosia or socially deficient communication may also be an early sign of frontal lobe dysfunction in DS and may represent a striking change from previous well-developed social capacities in the disorder (Nelson et al., 1995). Thus, executive dysfunction may be an early sign of aging and progression to dementia in DS. There are several studies describing magnetic resonance structural and/or volumetric differences and changes with age and dementia in DS (Beacher et al., 2009; Krasuski et al., 2002; Pinter et al., 2001; Roth et al., 1996; Teipel et al., 2004). Interestingly, frontal cortex volumes do not appear to decrease with AD in DS (Beacher et al., 2009) although volumes do decline with increasing age in those without dementia (Teipel et al., 2004). Further positron emission tomography imaging using amyloid beta (A $\beta$ )-ligands such as Pittsburgh Compound B (PiB) (Landt et al., 2011) or 2-(1-\{6-[(2- 
[fluorine-18] fluoroethyl)(methyl)amino]-2-naphthyl\}-ethylidene)malononitrile (FDDNP) (Nelson et al., 2011) show increased frontal binding after the age of 36 years (Landt et al., 2011).

Thus, the goal of the study was to specifically test the hypothesis that frontal dysfunction (measured by white matter integrity) would distinguish DS from non-DS and DS without dementia from those with dementia. Therefore, we measured WM integrity using FA with the prediction that frontal WM tracts would be particularly vulnerable to the presence of dementia in adults with DS and may also be compromised in DS relative to non-DS controls. We predicted that frontal white matter integrity would be associated with dementia in adults with DS. Therefore, we compared persons with DS and DS with clinically diagnosed dementia. These data provide an initial assessment of white matter integrity and form the basis of our efforts to evaluate longitudinal change in FA as an indicator of dementia evolution in DS.

\section{Methods}

\subsection{Participants}

Participants in this study were community residing men and women with DS, older than 35 years, recruited through local DS support groups and residential facilities in Kentucky and southern Ohio, into a longitudinal study of adult DS focused on evaluating decline in executive functioning and neural integrity as predictors of the development of dementia. Age- and gender-matched non-DS control participants provided medical history to document the absence of significant neurologic, cardiovascular and psychiatric disorders. At the time that these analyses were performed, we had 34 DS participants in the study. Four were excluded as they either could not be scanned because of a fear of the magnetic resonance imaging (MRI) scanner or there was too much motion in the MRI unit. Two participants were removed because of past traumatic brain injury or stroke. One participant was physically too large to be scanned. Two subjects had medical devices, which prevented an MRI scan. Thus, of 34 participants, 25 were able to be scanned, reflecting $73.5 \%$ of our cohort. Of the remaining 25, we had 10 demented adults with DS and thus we selected 10 age- and sex-matched nondemented DS participants to match to the 10 demented DS persons. Last, we recruited age and sex matched non-DS controls $(n=10)$. As a result, the final study cohort included 10 non-DS controls (CTL), 10 adults with DS (DS without dementia), and 10 DSAD (DS with AD) participants.

DS volunteers with active and unstable medical conditions (e.g., cardiovascular complications) were not included in the current sample for analysis. Thyroid dysfunction is common in individuals with DS, thus these participants were included if their thyroid dysfunction was medically controlled (DS $n=3$; DSAD $n=1$ ). Dementia diagnosis was determined through an expert consensus review of each participant with DS that involved 2 neurologists and 2 neuropsychologists using NINCDS-ADRDA criteria for dementia (McKhann et al., 1984) and included all data from medical history, medical and neurologic examinations, laboratory tests, structural imaging, mental status measures, and informant report of any changes in functional status and activities of daily living (McKhann, 1984). In addition to the objective mental status measures we obtained Dementia Questionnaire for 
Persons with Mental Retardation ratings from informants for each participant with DS (Evenhuis, 1996). Further, premorbid levels of functioning were derived from individual case files of existing academic and psychological test records, medical records, as well as family member interviews. Based on this information participants were categorized as low, medium, and high functioning based upon their pre-dementia level of functioning (Lott et al., 2011). Duration of dementia was established through medical records and family and/or caregiver interviews (Table 1).

All participants completed informed consent or assent (guardian consent). The study and research procedures were approved by the University of Kentucky Institutional Review Board.

\subsection{Neurocognitive measures}

All participants with DS completed medical, cognitive assessments, and DTI measurements obtained on the same day. The Brief Praxis Test (BPT; Dalton and Fedor, 1997) and the Severe Impairment Battery (SIB; Panisset et al., 1994) were used as neuropsychological measures for the present study. Both measures have been demonstrated to show progressive decline with worsening dementia in DS (Lott et al., 2012). Participant demographics and performances on the BPT and SIB for DS and DSAD groups are shown in Table 1. Levels of premorbid functioning did not differ between the participants with DS and DSAD (Fisher exact test $p=0.45$ ) as the sample reflected a 50 of $50 \%$ split of participants in the mild and moderate ranges overall and a 20 of $30 \%$ split for those persons diagnosed with dementia. The duration of dementia in DS adults with $\mathrm{AD}$ ranged from 0.9 to 6.0 years (median $=3.95$ years). Because of the minimal range and the sample size, we did not include this in the analysis of FA.

\subsection{Imaging}

All images were acquired on a 3T TIM Siemens scanner at UK Magnetic Resonance Imaging and Spectroscopy Center. DTI used an axial double refocused spin echo, echo planar imaging (EPI) sequence (repetition time $=8000 \mathrm{~ms}$, echo time $=96 \mathrm{~ms}$, field of view $=224 \mathrm{~mm}$, 52 slices, $2 \mathrm{~mm}$ isotropic resolution). The DTI images were acquired with 64 noncollinear encoding directions $\left(\mathrm{b}=1000 \mathrm{~s} / \mathrm{mm}^{2}\right)$ and 6 images without diffusion weighting $\left(\mathrm{b}=0 \mathrm{~s} / \mathrm{mm}^{2}, \mathrm{~b} 0\right)$.

DTI data were analyzed using FSL v4.1.5 (Functional MRI of the Brain software library, FMRIB). Raw images were pre-processed to correct for motion and residual eddy current distortion using a 12-parameter affine alignment to the corresponding b0 image via FMRIB linear image registration tool (http://www.fmrib.ox.ac.uk/fsl). Next, FMRIB's Diffusion Toolbox (FDT v2.0) was used to fit the diffusion tensor and calculate FA eigenvalues.

Registration of FA images into MNI152 space and subsequent voxelwise analyses followed a series of procedures known as tract-based spatial statistics (TBSS v1.2; http:// www.fmrib.ox.ac.uk/fsl/tbss/) as described in detail in our previous work (Gold et al., 2010a, 2010b). Briefly, all subjects' FA images were aligned to a common target using a nonlinear registration approach and then affine registered and resampled to $1 \mathrm{~mm}^{3} \mathrm{MNI} 152$ 
space. A mean FA image was used to create a common WM tract skeleton, which was then thresholded at an FA value of 0.2 to minimize partial volume effects after warping across subjects. Each participant's aligned FA image was subsequently projected onto the FA skeleton to account for residual misalignments between participants after the initial nonlinear registration.

This resulted in a common tract skeleton for each subject's FA image, which was filled with each participant's FA values from the centers of the nearest relevant tracts. The data are therefore in the form of a sparse 4D image with the fourth dimension being subject ID, which is treated as a random factor (Smith et al., 2006).

All voxelwise statistical analyses were performed via a permutation-based inference for nonparametric statistical thresholding using FSL's "randomize" (Nichols and Holmes, 2002). The permutation nonparametric tests used 5000 iterations and threshold-free cluster enhancement to avoid the use of an arbitrary threshold in the initial cluster formation. Unless otherwise note, all randomize analyses were corrected for multiple comparisons. Results are presented in a standard format on axial slices for comparison with other studies and projected onto a 3D transparent brain for more coherent visualization.

A 1-way analysis of variance was used to look for FA difference between the 3 groups (CTL, DS, DSAD) at $p<0.05$. We used 2 approaches for each analysis, which was to include age as a covariate and to not consider age in the analysis of variance. Overall, there were no differences in the statistical outcomes from these 2 approaches and thus we present the analysis with age as a covariate. Significant voxels from the omnibus test were then used to perform post hoc between group comparisons of FA values within the tract skeleton using 2 -sample $t$ tests. To correct for group permutations, all post hoc between group $t$ tests were thresholded at $p<0.01$. The first set of post hoc voxelwise analyses compared the $10 \mathrm{DS}$ subjects and 10 age- and gender-matched controls (Analysis 1). To detect differences in WM integrity as a function of dementia in DS, the second set of post hoc voxelwise analyses compared the 10 DS and 10 age- and gender-matched DSAD individuals (Analysis 2).

To determine the association between WM integrity and cognition, a third set of analyses used voxelwise correlations of FA and the BPT and SIB scores from all DS participants (20 subjects total) to provide sufficient power (Analysis 3). No BPT or SIB scores were available for CTL participants. A mean FA value for each participant was generated from all significant regions of interest (ROIs) resulting from Analysis 3.

To observe and detect associations between FA and the BPT and SIB for DS and DSAD separately (Analysis 4), a 2-tailed Spearman correlation corrected for age and gender was performed using the average FA from significant ROIs from Analysis 3. An overlay of the significant ROIs from analyses 1 and 2, the JHU DTI-based white-matter atlas (Zhang et al., 2005), and the Montreal Neurological Institute structural atlas (Grabner, 2006) was used to localize significant voxels within the major fiber tracts and the nearest brain lobe. 


\section{Results}

\subsection{Reduced WM integrity in nondemented DS adults relative to age- and gender-matched controls}

There were no significant voxels in the post hoc analysis for increased FA $(p<0.01)$ in DS compared with CTL. Figs. 1 and 2 show a post hoc analysis for decreased FA $(p<0.01)$ in DS compared with CTL (blue-Analysis 1). These regions include the anterior portions of the inferior fronto-occipital fasciculus (IFOF), superior longitudinal fasciculus (SLF), forceps minor (FMin), thalamic radiations (TR), frontal and temporal portions of the uncinate fasciculus (Un), inferior longitudinal fasciculus (ILF), and the cingulum (Cin). Table 2 lists the number of significant voxels within major fiber bundles and the cortical regions most closely associated with these WM tracts. The largest numbers of significantly lower FA voxels are in the frontal lobe when comparing DS with CTL.

\subsection{WM integrity is decreased in demented adults with DS compared with DS adults without dementia}

The DS and DSAD groups post hoc analyses yielded no significant differences when corrected for multiple comparisons at the $p<0.01$ level. However, to explore the possibility of more subtle differences between these 2 groups, we repeated these analyses using an uncorrected threshold $p<0.001$ and a cluster threshold of $8 \mathrm{~mm}^{3}$. There were no significant clusters for DS having lower FA than DSAD. However, there were significant clusters for DS having greater FA than DSAD. Results are presented in Figs. 1 and 2 (green-Analysis 2). Reductions in FA in DSAD participants versus DS participants were observed in: (1) symmetric regions $(Z=-10)$ containing the Un, FMin, and anterior Cin; (2) left frontal regions $(Z=7)$ containing the FMin, IFOF, SLF, Un, Cin, and anterior thalamic radiations (ATR); (3) symmetric regions $(Z=7)$ containing the fornix (cres) and/or stria terminalis and the ATR; and (4) symmetric regions $(Z=19)$ of the splenium containing colossal fibers and the IFOF, FMaj, SLF, and Cin.

\subsection{Reduced FA is associated with poorer cognition in DS}

No significant voxels for increased FA associated with BPT scores for DS participants with and without dementia. Areas where significant correlations of decreased FA associated with decreased BPT performance are shown (Figs. 1 and 2; red-Analysis 3). Lower BPT scores correlated with lower FA $(p<0.01)$ values in $(1)$ the anterior aspects of the body and posterior aspects of the genu of the corpus callosum $(Z=13,19,24)$; (2) the frontal lobe portions of the Cin, FMin, ATR, and Un $(Z=6,10,19)$; (3) the splenium of the corpus callosum ( $Z=13,19$, and 24); and (4) the posterior aspects of the forceps major (FMaj), IFOF, SLF, and ILF ( $Z=13,19$, and 24). Table 3 lists these and other significant voxels within major fiber bundles and their nearest brain lobes. No correlations with total SIB scores were found.

To characterize the strength of the association between BPT and average FA in DS, a 2tailed Spearman correlation correcting for age and gender was used. Higher BPT scores (indicating better cognitive function) correlated with higher FA scores (suggesting higher WM integrity; $\mathrm{r}=0.83, \mathrm{n}=20, p<0.001$; Fig. 3). 


\section{Discussion}

These DTI data are the first published investigation of aging and dementia in DS. We observed significantly lower FA in a subset of WM tracts between adults with DS compared with similar aged non-DS controls. Many of the FA decreases we detected involved frontal circuits. Further reductions in FA were seen in demented DS adults compared with DS adults without AD. Decreased FA in DS also was associated with BPT performance suggesting a functional link between frontal executive function, but not with global cognitive status (SIB).

The results of the present study suggest that 35 to 65 -year-old adults with DS have significantly lower WM integrity than similar aged non-DS controls, particularly in the frontal lobes. It is unclear if this is inherent to DS persons at all ages or if this is unique to middle aged and older DS adults. Previous imaging studies have shown reduced frontal lobe volume in DS in children and adults without dementia (Teipel et al., 2004), suggesting a strong developmental contribution to the present findings in nondemented DS adults. Our results are also consistent with prior reports of lower MRI-based frontal volume (Teipel et al., 2004) and lower MRI-based WM volumes in DS adults compared with non-DS controls (White et al., 2003).

The number of trending regions from the preliminary comparison of DSAD compared with nondemented DS in the present study suggests that DTI may contribute to our understanding of the mechanisms underlying dementia development in DS, although developmental reductions in frontal cortex FA may precede dementia leaving this region more vulnerable to aging and AD in DS. Reduced frontal lobe volumes are also consistent with the earliest manifestations of dementia in DS that include changes in personality and behavior, pragnosia, and socially deficient communication (Ball et al., 2008). The DTI changes demonstrated in the present study might either reflect such cortical volume loss or be a precursor to such change. We can speculate that the DTI connectivity differences seen in this study may therefore be associated with the reported striking change from previous well developed social capacities in DS with the development of dementia (Nelson et al., 1995). Data regarding this hypothesis should emerge from this project as longitudinal scans and behavioral changes are collected.

The BPT involves 20 simple tasks to assess limb praxis (armand leg movements), upper fine motor skills (opening a jar, picking up coins with each hand), and other highly practiced movements to simple verbal commands as well as imitation. Therefore, it is assumed to measure verbal comprehension and motor coordination and has been used in AD antioxidant clinical trials as a primary outcome measure (Lott et al., 2011; Matsuoka, et al., 2009). The impaired ability to carry out motor activities despite intact motor function is a hallmark symptom of most diagnostic criteria for dementia and as knowledge of overlearned actions is part of the BPT assessment; memory dysfunction likely also contributes to lower BPT scores to some extent.

Research on disorders of praxis has generally involved patients with stroke and several conceptual models of praxis have been proposed (for reviews see Koski et al., 2002; 
Petreska et al., 2007). Key elements of these proposed brain models suggest that deficits in praxis would result from the disconnection of parietal from frontal areas as well as temporal regions involved in comprehension. In reviews by Koski et al. (2002) as well as Petreska et al. (2007), areas such as the left dorsolateral frontal, left parietal, intraparietal sulcus, and the middle frontal gyrus (among other regions) have been implicated as important regions subserving praxis. In contrast, the SIB scale incorporates some praxis tasks but this only reflects $12 \%$ of the scale. Other SIB items focus on language production and comprehension $(46 \%)$ as well as memory (14\%) and orientation (8\%). While there were no statistically significant associations between FA and the SIB, it is possible that this reflects either the sample size, range of dementia severity, or the SIB's inclusion of more cortical functions.

The significant correlations between the BPT and FA in this study, particularly in frontal circuits, support the suggestion by Lott et al. (2012) that this test is sensitive to functional declines because of dementia in DS. A meta-analysis by Sexton et al., 2011 on DTI in mild cognitive impairment and $\mathrm{AD}$ shows widespread reductions in $\mathrm{FA}$ in the brain with the most notable and robust regions being the frontal and temporal lobes, posterior cingulum, corpus callosum, SLF, and Un. All these regions except the mid temporal regions were affected in the present study of DS when the BPT scores were correlated with FA, suggesting that dementia in DS has some similarities to dementia in the general population. The BPT correlates well with WM decline in middle aged and older adults with DS, and is particularly prominent in DS adults with dementia. Correlations between BPT and WM integrity include multiple brain regions, thus are widespread and consistent with regional WM declines in sporadic AD in the general population. Interestingly, there was overlap in the regions of the brain that showed reduced FA in DS as compared with age-matched control and areas in the DS brain associated with BPT scores. These included portions of the frontal and parietal callosal tracts. In the frontal lobes specifically there was an overlap in forceps minor, which connects some homologous anterior frontal lobe structures. In the parietal area, the main overlap was in splenium of corpus callosum, which connects some homologous parietal structures. These connections are consistent with the BPT data because coordination of signals between homologous frontal regions and homologous parietal regions would be expected to aid planning movements and spatial relations.

Losses in WM integrity could in part reflect the influence of AD-related pathology on WM itself. Accumulating evidence suggests a potential link between WM integrity and measures of AD pathology. For example, $A \beta$ deposits have been shown to be cytotoxic to oligodendrocytes in vitro (Xu et al., 2001) and increased levels of A $\beta$ peptides have been associated with reduced levels of myelin biochemical markers at autopsy in patients with $\mathrm{AD}$ (Roher et al., 2002). The aggregation of abnormally hyperphosphorylated tau may also affect WM microstructure given that tau is predominantly found in axons and is believed to bind to and stabilize microtubules, which are essential for structural integrity axonal transport (Shahani and Brandt, 2002). Myelin degeneration has also been reported in DS autopsy cases detected by electron microscopy in individuals as young as 21 years (Mattiace et al., 1991). The amount of frontal WM myelin basic protein correlated with neuropsychological test scores, including measures of frontal function (Wang et al., 2004). In DS, abnormalities in myelin basic protein may be related to several genes that are 
overexpressed in DS such as DSCAM, which regulates myelination (Saito et al., 2000). The enhanced vulnerability of the frontal WM in DS to a loss of integrity with age supports the hypothesis that late myelinating pathways may be particularly vulnerable to age and AD in DS, and that this may serve as a model for similar changes seen in late-onset sporadic AD in the general population (Stricker et al., 2009).

An additional possible contributor to reduced FA in DS and with dementia is vascular brain injury. In the present study, it is striking that there are significant white matter differences with a somewhat periventricular distribution (Fig. 2) in addition to the frontal connections and a similar pattern has been reported previously in non-DS cohorts. A recent study related white matter lesions and DTI in an autopsy cohort with AD. Ex vivo imaging of autopsy brain showed an overlap between FA and white matter lesions that reflect vascular deficits (Back et al., 2011). White matter hyperintensities, typically prominent in periventricular regions leads to reduced FA (Chao et al., 2013). Thus, it is entirely possible that some of the WM integrity losses observed in the present study are related to vascular insufficiency or injury, further longitudinal studies may help to elucidate this as a critical factor contributing to white matter integrity losses. Indeed, longitudinal studies of FA in DS adults as they transition to dementia may provide important insights into vascular contributors to white matter integrity and cognition. In turn, findings in DS, where dementia is more strongly ageassociated, may provide novel insights into the role of vascular integrity early in disease progression in $\mathrm{AD}$ in the general population.

\section{Summary}

The present study was based upon a relatively small sample size and our results to some extent are exploratory. However, our results suggest that WM integrity decline in DS because of AD may be similar to the declines observed in the general population with sporadic AD but with an increased impact on the frontal lobe circuitry in the DS population. These results suggest that WM degeneration may be a mechanistic basis for some of the cognitive changes observed before the development of overt cortical atrophy in DSAD as cognitive function is likely to depend, in part, on the microstructural properties of WM pathways connecting distributed cortical regions. Further work elucidating the temporal relationship of reduced WM integrity, cortical atrophy, and the development of dementia in adult DS is needed, and may provide insights useful for the development of biomarkers not only for the development of DSAD but for the global health threat of AD in the general population as well.

\section{Acknowledgments}

The authors are grateful to the participants and their families for enrolling in the study. We appreciate assistance in the study provided by Drs Erin Abner and Richard Kryscio for database support and Dr Brandon Davis and Dr Amy Dowling at the University of Kentucky for assistance with the study. Editing assistance was graciously provided by Ms Paula Thomason at the Sanders-Brown Center on Aging. The authors also would like to thank the Down syndrome Association of Louisville Inc and the Down syndrome Association of Greater Cincinnati for helping with recruitment and outreach. Further, Jose de Leon, MD, Director, UK Research Office; Director, Residency Education, Eastern State Hospital, Allen Brenzel, MD Medical Director, Kentucky Department for Behavioral Health, Developmental and Intellectual Disabilities, Edward N. Maxwell, MD, UK Outpatient Child \& Adolescent Psychiatrist, and Harold Kleinert, Ed.D, Professor of Rehabilitation Sciences, Executive Director of the Human Development Institute provided assistance in the early phase of our study. Guidance regarding recruitment and cognitive testing was provided by Dr Ira Lott and Eric Doran at the University of California, Irvine. Research 
reported in this manuscript was supported by Eunice Kennedy Shriver National Institute of Child Health and Development of the National Institutes of Health under award number R01HD064993. The content is solely the responsibility of the authors and does not necessarily represent the official views of the National Institutes of Health.

\section{References}

Aylward EH, Burt DB, Thorpe LU, Lai F, Dalton A. Diagnosis of dementia in individuals with intellectual disability. J. Intellect. Disabil. Res. 1997; 41(Pt 2):152-164. [PubMed: 9161927]

Back SA, Kroenke CD, Sherman LS, Lawrence G, Gong X, Taber EN, Sonnen JA, Larson EB, Montine TJ. White matter lesions defined by diffusion tensor imaging in older adults. Ann. Neurol. 2011; 70:465-476. [PubMed: 21905080]

Ball SL, Holland AJ, Treppner P, Watson PC, Huppert FA. Executive dysfunction and its association with personality and behaviour changes in the development of Alzheimer's disease in adults with Down syndrome and mild to moderate learning disabilities. Br. J. Clin. Psychol. 2008; 47(Pt 1):129. [PubMed: 17681112]

Basser PJ, Pajevic S, Pierpaoli C, Duda J, Aldroubi A. In vivo fiber tractography using DT-MRI data. Magn. Reson. Med. 2000; 44:625-632. [PubMed: 11025519]

Beacher F, Daly E, Simmons A, Prasher V, Morris R, Robinson C, Lovestone S, Murphy K, Murphy DG. Alzheimer's disease and Down's syndrome: an in vivo MRI study. Psychol. Med. 2009; 39:675-684. [PubMed: 18667098]

Bucur B, Madden DJ, Spaniol J, Provenzale JM, Cabeza R, White LE, Huettel SA. Age-related slowing of memory retrieval: contributions of perceptual speed and cerebral white matter integrity. Neurobiol. Aging. 2008; 29:1070-1079. [PubMed: 17383774]

Chao LL, Decarli C, Kriger S, Truran D, Zhang Y, Laxamana J, Villeneuve S, Jagust WJ, Sanossian N, Mack WJ, Chui HC, Weiner MW. Associations between white matter hyperintensities and beta amyloid on integrity of projection, association, and limbic fiber tracts measured with diffusion tensor MRI. PLoS One. 2013; 8:e65175. [PubMed: 23762308]

Cooper SA, Prasher VP. Maladaptive behaviours and symptoms of dementia in adults with Down's syndrome compared with adults with intellectual disability of other aetiologies. J. Intellect. Disabil. Res. 1998; 42(Pt 4):293-300. [PubMed: 9786444]

Dalton, A.; Fedor, B. NYS Institute for Basic Research in Developmental Disabilities. New York: Staten Island; 1997. DYSPRAXIA Scale for Adults with Down Syndrome.

Evenhuis HM. Further evaluation of the dementia questionnaire for persons with mental retardation (DMR). J. Intellect. Disabil. Res. 1996; 40:369-373. [PubMed: 8884592]

Gold BT, Powell DK, Andersen AH, Smith CD. Alterations in multiple measures of white matter integrity in normal women at high risk for Alzheimer's disease. Neuroimage. 2010a; 52:14871494. [PubMed: 20493952]

Gold BT, Powell DK, Xuan L, Jicha GA, Smith CD. Age-related slowing of task switching is associated with decreased integrity of frontoparietal white matter. Neurobiol. Aging. 2010b; 31:512-522. [PubMed: 18495298]

Grabner G. Symmetric atlasing and model based segmentation: an application to the hippocampus in older adults. Med. Image Comput. Comput. Assist. Interv. 2006; 4191:9.

Head E, Lott IT, Patterson D, Doran E, Haier RJ. Possible compensatory events in adult Down syndrome brain prior to the development of Alzheimer disease neuropathology: targets for nonpharmacological intervention. J. Alzheimers Dis. 2007; 11:61-76. [PubMed: 17361036]

Holland AJ, Hon J, Huppert FA, Stevens F. Incidence and course of dementia in people with Down's syndrome: findings from a population-based study. J. Intellect. Disabil. Res. 2000; 44(Pt 2):138146. [PubMed: 10898377]

Koski L, Iacoboni M, Mazziotta JC. Deconstructing apraxia: understanding disorders of intentional movement after stroke. Curr. Opin. Neurol. 2002; 15:71-77. [PubMed: 11796953]

Krasuski JS, Alexander GE, Horwitz B, Rapoport SI, Schapiro MB. Relation of medial temporal lobe volumes to age and memory function in nondemented adults with Down's syndrome: implications for the prodromal phase of Alzheimer's disease. Am. J. Psychiatry. 2002; 159:74-81. [PubMed: 11772693] 
Landt J, D'Abrera JC, Holland AJ, Aigbirhio FI, Fryer TD, Canales R, Hong YT, Menon DK, Baron JC, Zaman SH. Using positron emission tomography and carbon 11-labeled Pittsburgh compound B to image brain fibrillar beta-amyloid in adults with down syndrome: safety, acceptability, and feasibility. Arch. Neurol. 2011; 68:890-896. [PubMed: 21403005]

Lott IT. Neurological phenotypes for Down syndrome across the life span. Prog. Brain Res. 2012; 197:101-121. [PubMed: 22541290]

Lott IT, Doran E, Nguyen VQ, Tournay A, Head E, Gillen DL. Down syndrome and dementia: a randomized, controlled trial of antioxidant supplementation. Am. J. Med. Genet. A. 2011; 155A: 1939-1948. [PubMed: 21739598]

Lott IT, Doran E, Nguyen VQ, Tournay A, Movsesyan N, Gillen DL. Down syndrome and dementia: seizures and cognitive decline. J. Alzheimers Dis. 2012; 29:177-185. [PubMed: 22214782]

Madden DJ, Bennett IJ, Burzynska A, Potter GG, Chen NK, Song AW. Diffusion tensor imaging of cerebral white matter integrity in cognitive aging. Biochim. Biophys. Acta. 2012; 1822:386-400. [PubMed: 21871957]

Madden DJ, Bennett IJ, Song AW. Cerebral white matter integrity and cognitive aging: contributions from diffusion tensor imaging. Neuropsychol. Rev. 2009; 19:415-435. [PubMed: 19705281]

Matsuoka Y, Andrews HF, Becker AG, Gray AJ, Mehta PD, Sano MC, Dalton AJ, Aisen PS. The relationship of plasma abeta levels to dementia in aging individuals with Down syndrome. Alzheimer Dis. Assoc. Disord. 2009; 23:315-318. [PubMed: 19571732]

Mattiace LA, Kress Y, Davies P, Ksiezak-Reding H, Yen SH, Dickson DW. Ubiquitinimmunoreactive dystrophic neurites in Down's syndrome brains. J. Neuropathol. Exp. Neurol. 1991; 50:547-559. [PubMed: 1654399]

McKhann G, Drachman D, Folstein M, Katzman R, Price D, Stadlan EM. Clinical diagnosis of Alzheimer's disease: report of the NINCDS-ADRDA work group under the auspices of Department of Health and Human Services task force on Alzheimer's disease. Neurology. 1984; 34:939-944. [PubMed: 6610841]

Nelson L, Lott I, Touchette P, Satz P, D’Elia L. Detection of Alzheimer disease in individuals with Down syndrome. Am. J. Ment. Retard. 1995; 99:616-622. [PubMed: 7632429]

Nelson LD, Siddarth P, Kepe V, Scheibel KE, Huang SC, Barrio JR, Small GW. Positron emission tomography of brain beta-amyloid and tau levels in adults with Down syndrome. Arch. Neurol. 2011; 68:768-774. [PubMed: 21670401]

Nichols TE, Holmes AP. Nonparametric permutation tests for functional neuroimaging: a primer with examples. Hum. Brain Mapp. 2002; 15:1-25. [PubMed: 11747097]

Panisset M, Roudier M, Saxton J, Boller F. Severe impairment battery. A neuropsychological test for severely demented patients. Arch. Neurol. 1994; 51:41-45. [PubMed: 8274108]

Petreska B, Adriani M, Blanke O, Billard AG. Apraxia: a review. Prog. Brain Res. 2007; 164:61-83. [PubMed: 17920426]

Pfefferbaum A, Sullivan EV. Increased brain white matter diffusivity in normal adult aging: relationship to anisotropy and partial voluming. Magn. Reson. Med. 2003; 49:953-961. [PubMed: 12704779]

Pinter JD, Eliez S, Schmitt JE, Capone GT, Reiss AL. Neuroanatomy of Down's syndrome: a highresolution MRI study. Am. J. Psychiatry. 2001; 158:1659-1665. [PubMed: 11578999]

Roher AE, Weiss N, Kokjohn TA, Kuo YM, Kalback W, Anthony J, Watson D, Luehrs DC, Sue L, Walker D, Emmerling M, Goux W, Beach T. Increased A beta peptides and reduced cholesterol and myelin proteins characterize white matter degeneration in Alzheimer's disease. Biochemistry. 2002; 41:11080-11090. [PubMed: 12220172]

Roth GM, Sun B, Greensite FS, Lott IT, Dietrich RB. Premature aging in persons with Down syndrome: MR findings. Am. J. Neuroradiol. 1996; 17:1283-1289. [PubMed: 8871713]

Saito Y, Oka A, Mizuguchi M, Motonaga K, Mori Y, Becker LE, Arima K, Miyauchi J, Takashima S. The developmental and aging changes of Down's syndrome cell adhesion molecule expression in normal and Down's syndrome brains. Acta Neuropathol. (Berl). 2000; 100:654-664. [PubMed: 11078217]

Schupf N, Sergievsky GH. Genetic and host factors for dementia in Down's syndrome. Br. J. Psychiatry. 2002; 180:405-410. [PubMed: 11983636] 
Sexton CE, Kalu UG, Filippini N, Mackay CE, Ebmeier KP. A meta-analysis of diffusion tensor imaging in mild cognitive impairment and Alzheimer's disease. Neurobiol. Aging. 2011; 32:2322.e5-2322.e18. [PubMed: 20619504]

Shahani N, Brandt R. Functions and malfunctions of the tau proteins. Cell Mol. Life Sci. 2002; 59:1668-1680. [PubMed: 12475178]

Smith SM, Jenkinson M, Johansen-Berg H, Rueckert D, Nichols TE, Mackay CE, Watkins KE, Ciccarelli O, Cader MZ, Matthews PM, Behrens TE. Tract-based spatial statistics: voxelwise analysis of multisubject diffusion data. Neuroimage. 2006; 31:1487-1505. [PubMed: 16624579]

Stricker NH, Schweinsburg BC, Delano-Wood L, Wierenga CE, Bangen KJ, Haaland KY, Frank LR, Salmon DP, Bondi MW. Decreased white matter integrity in late-myelinating fiber pathways in Alzheimer's disease supports retrogenesis. Neuroimage. 2009; 45:10-16. [PubMed: 19100839]

Teipel SJ, Alexander GE, Schapiro MB, Moller HJ, Rapoport SI, Hampel H. Age-related cortical grey matter reductions in non-demented Down's syndrome adults determined by MRI with voxel-based morphometry. Brain. 2004; 127:811-824. [PubMed: 14985261]

Wang DS, Bennett DA, Mufson EJ, Mattila P, Cochran E, Dickson DW. Contribution of changes in ubiquitin and myelin basic protein to age-related cognitive decline. Neurosci. Res. 2004; 48:93100. [PubMed: 14687885]

White NS, Alkire MT, Haier RJ. A voxel-based morphometric study of nondemented adults with Down syndrome. Neuroimage. 2003; 20:393-403. [PubMed: 14527599]

Wimberger DM, Roberts TP, Barkovich AJ, Prayer LM, Moseley ME, Kucharczyk J. Identification of “premyelination” by diffusion-weighted MRI. J. Comput. Assist Tomogr. 1995; 19:28-33. [PubMed: 7529780]

Wisniewski K, Wisniewski H, Wen G. Occurrence of neuropathological changes and dementia of Alzheimer's disease in Down's syndrome. Ann. Neurol. 1985; 17:278-282. [PubMed: 3158266]

Xu J, Chen S, Ahmed SH, Chen H, Ku G, Goldberg MP, Hsu CY. Amyloid-beta peptides are cytotoxic to oligodendrocytes. J. Neurosci. 2001; 21:RC118. [PubMed: 11150354]

Zhang JH, Sampogna S, Morales FR, Chase MH. Age-related ultrastructural changes in hypocretinergic terminals in the brainstem and spinal cord of cats. Neurosci. Lett. 2005; 373:171174. [PubMed: 15619537] 

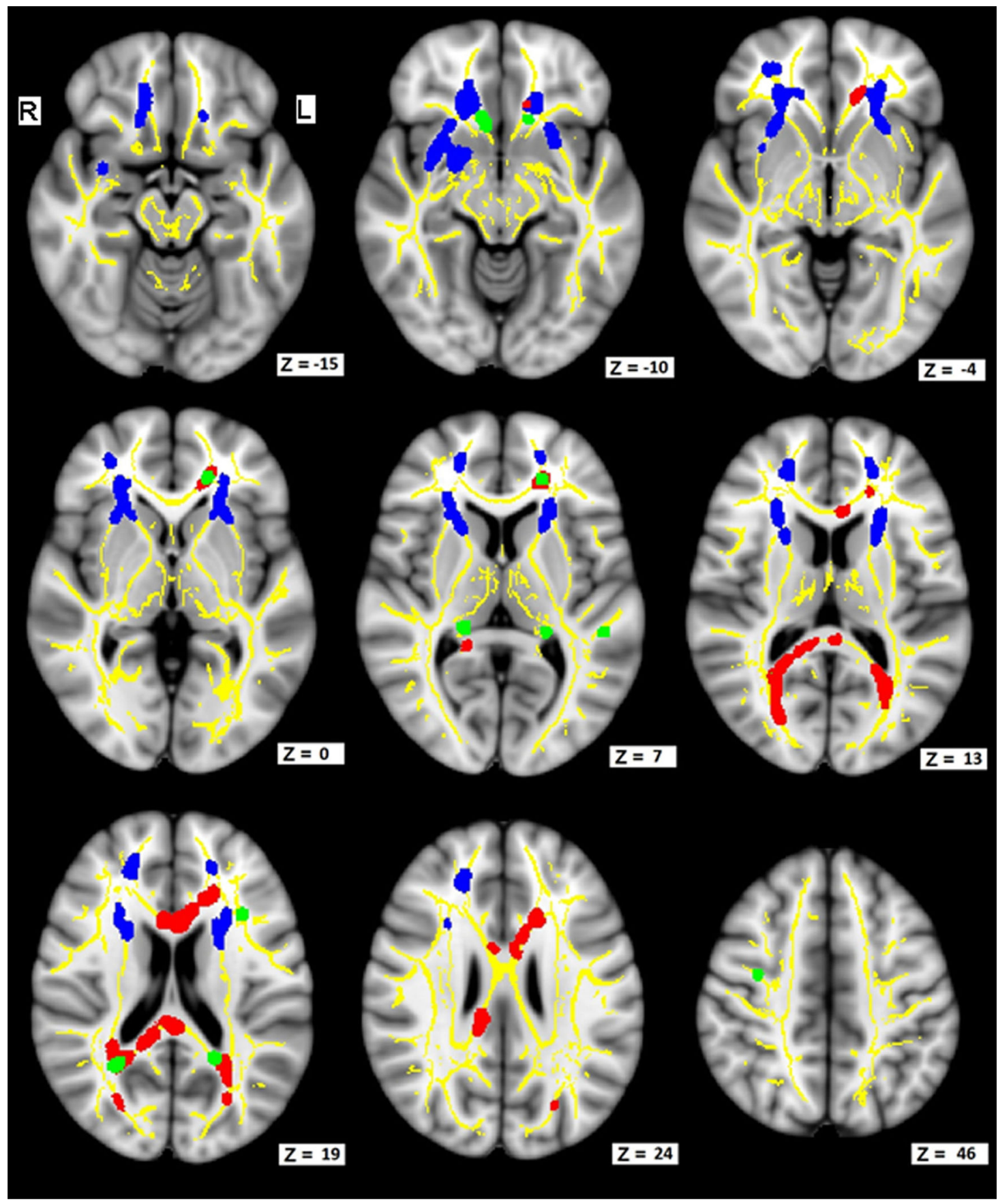

Fig. 1.

FA is lower in DS compared with controls without DS, and further reduced with dementia in DS (DSAD). FA decreases in DS are associated with lower BPT scores. Images show axial slices in MNI space and illustrate representative sampling of FA analyses. $\mathrm{Z}$ is the voxel coordinate in MNI 1-mm template space. Yellow illustrates the underlying FA skeleton common to all participants. Blue shows areas that had reduced FA when comparing nondemented DS adults with non-DS controls $(p<0.01$, corrected). Green shows areas where demented adults with DS have lower FA than nondemented adults with DS $(p<0.001$, uncorrected). Red shows areas where lower FA is associated with lower BPT scores. Abbreviations: BPT, Brief Praxis Test; DS, Down syndrome; DSAD, Down syndrome with dementia; FA, fractional anisotropy; MNI, Montreal Neurologic Institute. 


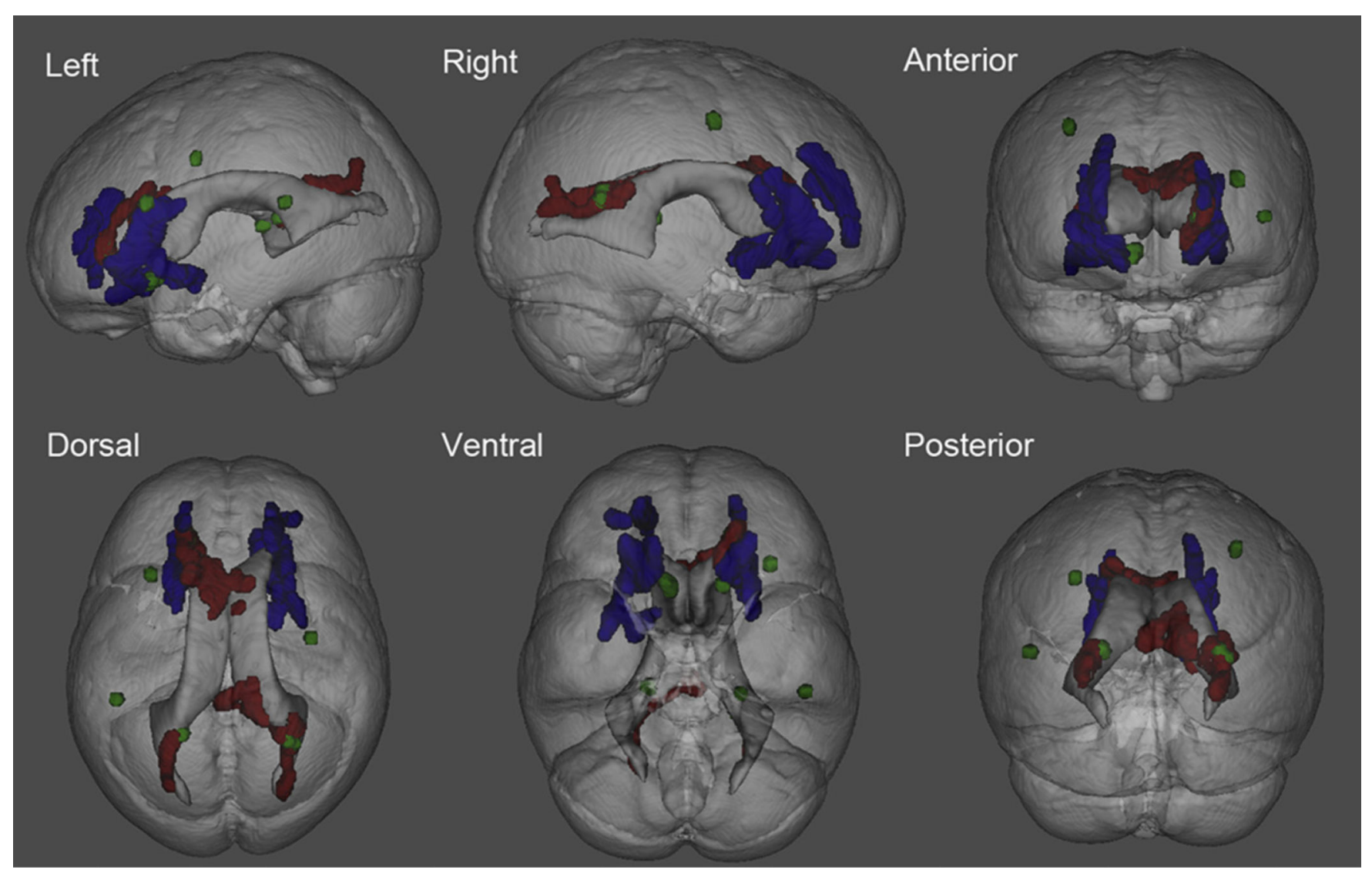

Fig. 2.

A 3D transparent view illustrating regions where FA is associated with DS, dementia and BPT scores. Blue shows areas that had reduced FA when comparing nondemented DS adults with non-DS controls $(p<0.01$ corrected). Green shows areas where demented adults with DS have lower FA than nondemented adults with DS $(p<0.001$, uncorrected). Red shows areas where lower FA is associated with lower BPT scores in DS adults. Abbreviations: BPT, Brief Praxis Test; DS, Down syndrome; FA, fractional anisotropy. 


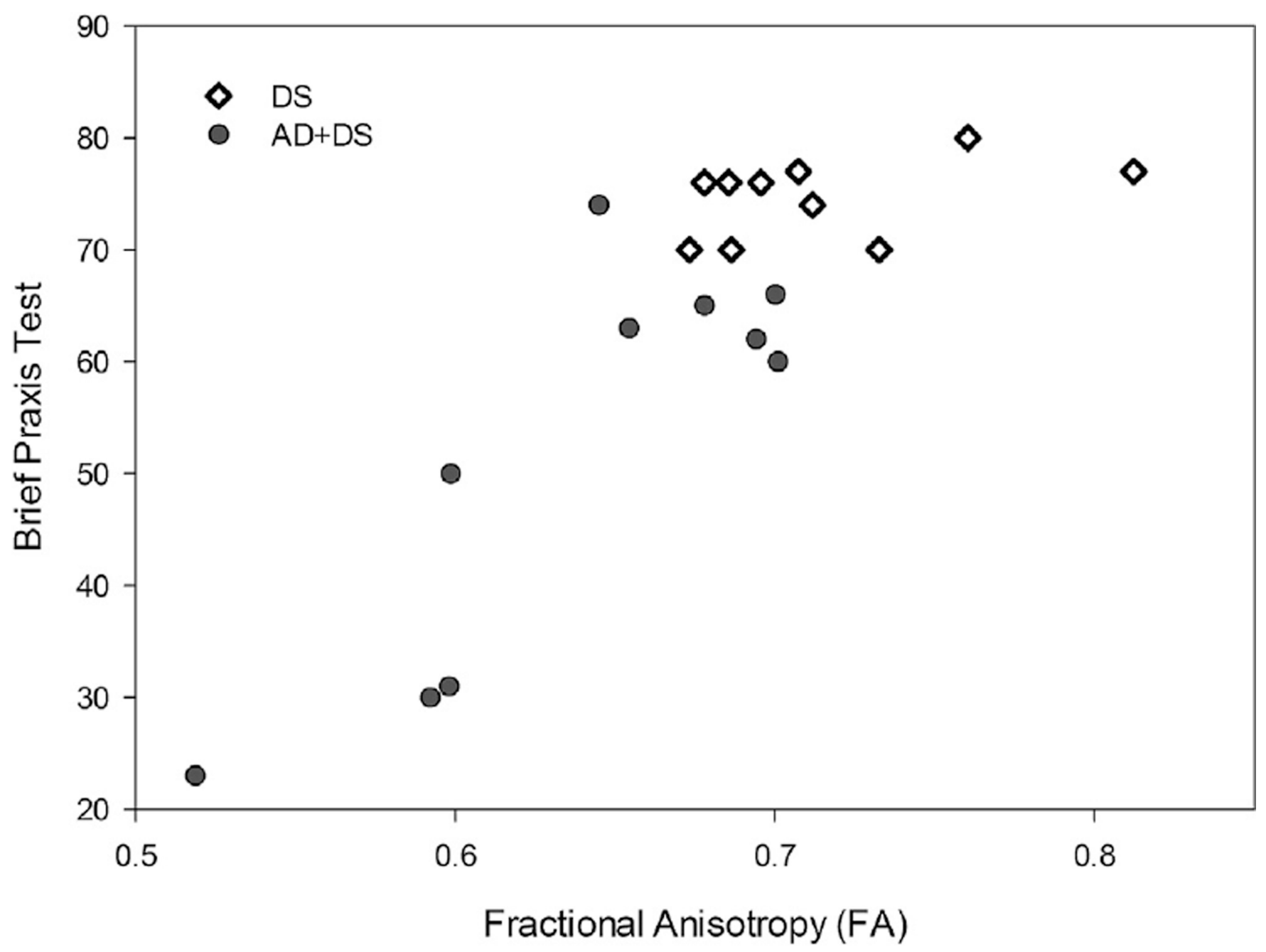

Fig. 3.

A graph illustrating the association between average brain FA from all the significant voxels from Analysis 3 (red in figures 1 and 2) and individual BPT scores in DS and DS with AD. FA is strongly correlated with BPT scores in demented individuals.

Abbreviations: AD, Alzheimer's disease; BPT, Brief Praxis Test; DS, Down syndrome; FA, fractional anisotropy. 


\section{Table 1}

Demographics of study participants

\begin{tabular}{|c|c|c|c|c|}
\hline Participant characteristics & All DS & DS & DSAD & CTL \\
\hline Age (mean, SD) ${ }^{a}$ & $51.38(6.48)$ & $50.61(5.53)$ & $52.16(7.54)$ & $51.07(2.14)$ \\
\hline Gender (men/women) & $6 / 14$ & $3 / 7$ & $3 / 7$ & $3 / 7$ \\
\hline $\mathrm{BPT}($ mean, SD) $b$ & $63.65(16.78)$ & $73.40(4.06)$ & $53.90(19.15)$ & - \\
\hline $\mathrm{SIB}(\text { mean, } \mathrm{SD})^{c}$ & $74 / 70(22.04)$ & $85.40(11.18)$ & $64.00(25.45)$ & - \\
\hline SIB Praxis (mean, $\mathrm{SD})^{d}$ & $15.75(5.06)$ & $18.30(2.21)$ & $13.10(5.88)$ & - \\
\hline $\operatorname{DMR}(\text { mean, } \mathrm{SD})^{e}$ & $19.30(16.49)$ & $6.70(3.52)$ & $31.90(14.44)$ & - \\
\hline \multirow[t]{2}{*}{ Dementia duration (y; mean, SD) } & - & - & $5.31(4.90)$ & - \\
\hline & & & median $=4.15$ & \\
\hline
\end{tabular}

Key: BPT, Brief Praxis Test; CTL, non-DS controls, DMR, Dementia Questionnaire for Persons with Mental Retardation; DS, Down syndrome; DSAD, Down syndrome with dementia; SD, standard deviation; SIB, Severe Impairment Battery.

${ }^{a}$ Paired $t$ test comparisons between the DS and DSAD are not significant for age $(p=0.61)$. Significant differences are seen between DS groups.

$b_{\text {BPT }(p<0.01) .}$

$c_{\mathrm{SIB}}(p<0.05)$.

$d_{\text {SIB Praxis }}(p<0.05)$.

$e_{\mathrm{DMR}}(p<0.001)$ scores; dementia duration is based on onset of symptoms derived from clinical interviews. 
Table 2

Differences in FA between DS adults and similarly aged non-DS (CTL) participants

\begin{tabular}{|c|c|c|c|c|}
\hline \multicolumn{5}{|c|}{ FA-group analysis-DS versus CTL } \\
\hline & Frontal & Parietal & Temporal & Occipital \\
\hline Forceps-minor & 2119 & 0 & 0 & 0 \\
\hline IFOF-R & 1557 & 106 & 546 & 0 \\
\hline IFOF-R & 1718 & 42 & 410 & 0 \\
\hline Uncinate-L & 1342 & 59 & 125 & 0 \\
\hline Uncinate-R & 1031 & 15 & 90 & 0 \\
\hline Genu-CC & 175 & 0 & 0 & 0 \\
\hline Ant_Thal_Rad-L & 1519 & 60 & 15 & 1 \\
\hline Ant_Thal_Rad-R & 1124 & 0 & 4 & 0 \\
\hline SLF-L & 657 & 1176 & 64 & 0 \\
\hline SLF-R & 245 & 11 & 0 & 0 \\
\hline Cing_Cing Gyr-R & 199 & 266 & 0 & 74 \\
\hline Cing_Cing Gyr-L & 0 & 0 & 0 & 0 \\
\hline Body-CC & 0 & 7 & 0 & 0 \\
\hline Cing_Hipp-L & 0 & 0 & 0 & 0 \\
\hline Cing_Hipp-R & 0 & 0 & 0 & 0 \\
\hline CS-L & 8 & 118 & 0 & 0 \\
\hline CS-R & 0 & 0 & 0 & 0 \\
\hline Splenium-CC & 0 & 160 & 0 & 0 \\
\hline Forceps-major & 0 & 7 & 0 & 0 \\
\hline ILF-L & 0 & 92 & 473 & 39 \\
\hline ILF-R & 0 & 0 & 259 & 0 \\
\hline
\end{tabular}

Summary of $1 \mathrm{~mm}^{3}$ voxels within major white matter fiber tracks (corresponding to blue ROIs from Fig. 2). The number of voxels within each lobe of the brain is listed. Key: Ant_Thal_Rad, anterior thalamic radiations; CC, corpus callosum; Cing_Cing_-Gyr, cingulum of cingulate gyrus; Cing_Hipp, cingulum of hippocampus; CS, corticospinal; FA, fractional anisotropy; IFOF, inferior frontal occipital fasciculus; ILF, inferior longitudinal fasciculus; L, left; R, right; ROIs, regions of interest; SLF, superior longitudinal fasciculus. 
Table 3

Regions of interest that show a significant correlation between the BPT and FA in all DS participants, with and without dementia

\begin{tabular}{|c|c|c|c|c|}
\hline \multicolumn{5}{|c|}{ FA correlation-Brief praxis test in DS } \\
\hline & Frontal & Parietal & Temporal & Occipital \\
\hline Forceps-minor & 379 & 0 & 0 & 0 \\
\hline IFOF-R & 25 & 32 & 0 & 21 \\
\hline IFOF-R & 0 & 133 & 0 & 82 \\
\hline Uncinate-L & 27 & 0 & 0 & 0 \\
\hline Uncinate- $\mathrm{R}$ & 0 & 0 & 0 & 0 \\
\hline Genu-CC & 140 & 0 & 0 & 0 \\
\hline Ant_Thal_Rad-L & 83 & 4 & 0 & 1 \\
\hline Ant_Thal_Rad-R & 0 & 0 & 0 & 0 \\
\hline SLF-L & 0 & 0 & 0 & 0 \\
\hline SLF-R & 0 & 3 & 0 & 0 \\
\hline Cing_Cing_Gyr-R & 111 & 0 & 0 & 0 \\
\hline Cing_Cing_Gyr-L & 0 & 0 & 0 & 0 \\
\hline Body-CC & 269 & 29 & 0 & 0 \\
\hline Cing_Hipp-L & 0 & 0 & 0 & 0 \\
\hline Cing_Hipp-R & 0 & 6 & 0 & 0 \\
\hline CS-L & 0 & 0 & 0 & 0 \\
\hline CS-R & 0 & 0 & 0 & 0 \\
\hline Splenium-CC & 0 & 520 & 0 & 3 \\
\hline Forceps-major & 0 & 575 & 0 & 156 \\
\hline ILF-L & 0 & 18 & 0 & 16 \\
\hline ILF-R & 0 & 38 & 0 & 36 \\
\hline
\end{tabular}

Summary of $1 \mathrm{~mm}^{3}$ voxels within major white matter fiber tracks (corresponding to red ROIs from Fig. 2). The number of voxels within each lobe of the brain is listed. Key: Ant_Thal_Rad, anterior thalamic radiations; BPT, Brief Praxis Test; CC, corpus callosum; Cing_Cing_Gyr, cingulum of cingulate gyrus; Cing_Hipp, cingulum of hippocampus, CS, corticospinal, DS, Down syndrome; FA, fractional anisotropy; IFOF, inferior frontal occipital fasciculus; ILF, inferior longitudinal fasciculus; L, left; R, right; SLF, superior longitudinal fasciculus. 